\title{
Penfigoide ampolloso asociado a uso de vildagliptina. Reporte de caso
}

\section{Bullous pemphigoid associated with vildagliptin use. Case report}

\author{
Carlos H. Calderón-Franco ${ }^{*}$, Víctor Trujillo-Hernandez ${ }^{2}$, Santiago Campbell-Silva ${ }^{3}$, \\ Diego J. AlVis-PeÑA ${ }^{4}$ Y JULIETH GONZALEs-CeróN ${ }^{5}$
}

'Servicio de Medicina Interna e Investigación, Clínica Medilaser, Florencia-Caquetá; ${ }^{2}$ Unidad académica, Fundación Universitaria Ciencias de la Salud, Florencia-Caquetá; ${ }^{3}$ Servicio de Medicina Interna e Investigación, Clínica Medilaser, Florencia-Caquetá; ${ }^{4}$ Servicio de Medicina Interna e Investigación, ESE Hospital San Antonio de Timana, Pitalito-Huila; ${ }^{5}$ Servicio de Hematooncología e Investigación, Hospital Fundación Cardio Infantil, Bogotá. Colombia

\section{RESUMEN}

La vildagliptina es un fármaco inhibidor de la dipeptidil peptidasa 4 (iDPP-4) y dentro de sus reacciones medicamentosas se ha descrito la manifestación dermatológica del penfigoide ampolloso (PA), una enfermedad crónica poco común, autoinmune y subepidérmica. Presentamos el caso de un paciente con antecedente de diabetes tipo 2 , se encontraba en manejo con metformina, empagliflozina y vildagliptina; presentó lesiones vesiculopapulares con bases eritematosas, visualizadas sobre los brazos, piernas y tronco. Se suspendió el tratamiento hipoglucemiante, se inició terapia inmunosupresora y posterior al manejo inmunosupresor las lesiones dermatológicas mejoraron. Este reporte de caso es una base de documentación científica sobre PA en pacientes con vildagliptina.

Palabras clave: Penfigoide ampolloso. Hipoglucemiantes. Vildagliptina. Inhibidores de la dipeptidil peptidasa 4.

\begin{abstract}
Vildagliptin is a DPP-4 (iDPP-4) inhibitor drug. Among its drug reactions, the dermatological manifestation of bullous pemphigoid (BP), a chronic, rare, autoimmune and subepidermal disease, has been described. We present the case of a patient with antecedent diabetes type 2, who was under treatment with metformin, empaglifozin and vildagliptin; he presented vesicular papular lesions with erythematous bases, visualized on arms, legs and trunk. Hypoglycemic treatment was suspended, immunosuppressive therapy was started and after immunosuppressive management, dermatological lesions improved. This case report is a scientific documentation base on BP in patients with vildagliptin.
\end{abstract}

Key words: Pemphigoid. Bullous. Hypoglycemic Agents. Vildagliptin. Dipeptidyl-Peptidase IV Inhibitors.

\section{Correspondencia:}

*Carlos H. Calderón-Franco

E-mail: cacalderon190@gmail.com
Fecha de recepción: 15-09-2020

Fecha de aceptación: 20-11-2020

DOI: $10.24875 /$ RME. 20000090
Disponible en internet: 02-11-2021 Rev Mex Endocrinol Metab Nutr. 2021;8:202-7

2462-4144 / @ 2020 Sociedad Mexicana de Nutrición y Endocrinología, AC. Publicado por Permanyer. Este es un artículo open access bajo la licencia CC BY-NC-ND (http://creativecommons.org/licenses/by-nc-nd/4.0/). 


\section{INTRODUCCIÓN}

El penfigoide ampolloso (PA) inducido por medicamentos es una alteración inmunitaria adquirida, que se ha descrito en poblaciones etarias mayores de 60 años. Se caracteriza por erupción ampollosa pruriginosa generalizada que compromete la subepidermis y muy pocas veces altera las membranas de la mucosa, es una patología con gran dificultad para establecer el diagnóstico en fases tempranas de la enfermedad o en las presentaciones atípicas en las que no aparecen lesiones ampollosas completamente'. Estudios observacionales recientes han reportado un incremento en la incidencia de PA de 1.9 a 4.3 casos por 100,000 personas-año secundario al aumento de uso de algunos fármacos que incrementa el riesgo de desarrollarlo ${ }^{2}$. Diferentes estudios sugieren que el consumo de agentes orales como los inhibidores de dipeptidil peptidasa 4 (iDPP-4), fármacos utilizados para tratar la diabetes tipo 2, puede estar asociado al desarrollo de $\mathrm{PA}^{3,4}$.

El PA se ha descrito como una enfermedad inmunitaria asociada a respuesta humoral y celular enfocada contra autoantígenos bien diferenciados: el BP180, el cual es una proteína transmembrana con gran dominio extracelular colagenasa y el BP230, que es una proteína citoplasmática que pertenece a la familia de la plaquina ${ }^{1,5}$.

A continuación, reportamos un caso de PA en un paciente de sexo masculino de 70 años que ingresa al servicio de urgencia de un hospital de tercer nivel con lesiones vesiculopapulares y escoriaciones en diferentes áreas de la superficie corporal.

\section{PRESENTACIÓN DEL CASO}

Hombre de 70 años, de etnia mestizo, procedente y residente de Florencia, Caquetá (Colombia), de profesión médico, pero en la actualidad cesante. Ingresó al servicio de urgencias de un hospital de alta complejidad de Florencia debido a un cuadro clínico lento pero progresivo consistente en lesiones tipo vesículas pruriginosas, entre 0.5 y 2 milímetros

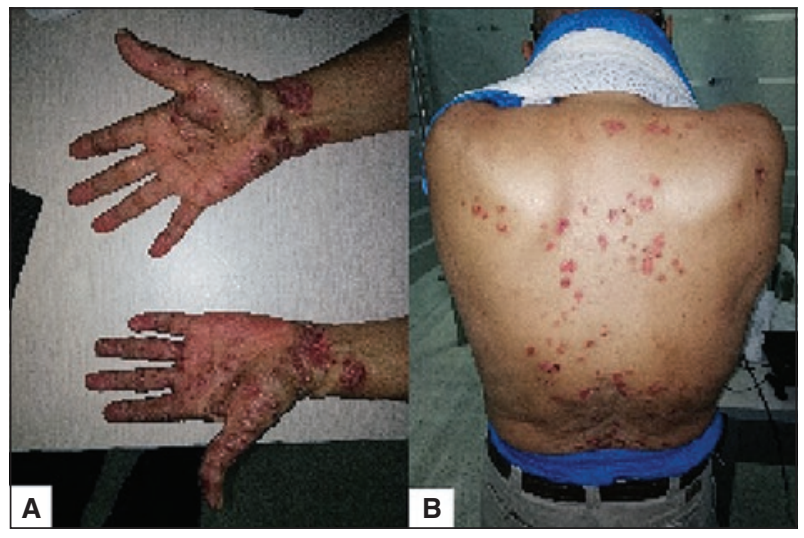

Figura 1. A: escoriaciones con lesiones vesiculares, papulares y costrosas sobre una base eritematosa en extremidades superiores. B: mismas lesiones dermatológicas ya descritas, pero en área dorsal y lumbar

de diámetro de contenido seroso, base eritematosa, en miembros superiores e inferiores (Fig. 1).

Dentro de sus antecedentes de importancia presenta hipertensión arterial, diabetes tipo 2, enfermedad renal crónica estadio 3B (39.13 ml/min CKD-EPI [Chronic Kidney Disease Epidemiology Collaboration]); negó antecedentes familiares y alérgicos, y dentro de los antecedentes farmacológicos mencionó: enalapril ( $20 \mathrm{mg}$ ), amlodipino (5 mg), hidroclorotiazida ( $25 \mathrm{mg}$ ), hipoglucemiantes con empagliflozina (25 mg), vildagliptina (50 mg) y metformina (500 mg).

El paciente refiere un manejo de más de 12 meses con hipoglucemiantes (metformina más empagliflozina); sin embargo, su médico especialista realizó un ajuste de tratamiento donde se agregó vildagliptina a la terapia médica, consolidando la triple terapia oral (metformina, empagliflozina y vildagliptina). El paciente argumentó aparición de lesiones de tipo dermatológicas días después del inicio de su nuevo régimen terapéutico, sintomatología que fue manejada por diferentes especialidades, entre estas dermatología y alergología, quienes ordenaron tratamiento con etanercep ante la sospecha de pitiriasis liquenoide; pero ante la persistencia de sus lesiones consultó al servicio de urgencias dos meses después del inicio de sus síntomas y del cambio de régimen terapéutico. El paciente en la revisión de sistemas negó alzas térmicas, afección de mucosas $\mathrm{y}$ adenomegalias. 
Al examen físico inicial se describe presión arterial en 134/84 mmHg, frecuencia cardiaca de 76 latidos por minuto, frecuencia respiratoria de 21 respiraciones por minuto, glucometría $170 \mathrm{mg} / \mathrm{dl}$, pulsioximetría del 98\% al ambiente, afebril y peso de $75 \mathrm{~kg}$, hidratado, sin ingurgitación venosa yugular, sin presencia de adenopatías, tórax normoexpansible, ruidos cardiacos rítmicos sin soplos, sin presencia de edemas, sistema neurológico sin alteraciones, en piel con presencia de lesiones tipo vesículas pruriginosas, entre 0.5 y 2 milímetros de diámetro de contenido seroso, base eritematosa, sin secreciones activas a nivel de manos, antebrazos y brazos de mayor predominio en zonas de flexión, así como en piernas, pies, tórax, abdomen y en el área dorsolumbar.

Los laboratorios al ingreso no presentaron alteraciones relevantes: hemoglobina $12.1 \mathrm{~g} / \mathrm{dl}$, hematocrito $34.4 \%$, recuento plaquetario $41110^{3} \times \mathrm{mm}^{3}$, leucocitos $9.7010^{3} \times \mathrm{mm}^{3}$, neutrófilos $80.1 \%$, creatinina $1.73 \mathrm{mg} / \mathrm{dl}$, glucosa sérica $185 \mathrm{mg} / \mathrm{dl}$, electrolitos en rango normal para el potasio y leve hiponatremia sin sintomatología clínica (sodio $130.5 \mathrm{mmol} / \mathrm{l}$, potasio $4.38 \mathrm{mmol} / \mathrm{l}$ ) con función hepática normal (aspartato aminotransferasa $16 \mathrm{U} / \mathrm{l}$; alanina aminotransferasa $20 \mathrm{U} / \mathrm{I})$.

Al no presentar alteraciones significativas en los laboratorios y por los hallazgos anteriores descritos se ordenó realización de biopsia de las lesiones, en donde los resultados histopatológicos mostraron una gran ampolla subepidérmica con infiltrados de predominio eosinofílico que se organizan a lo largo de la membrana basal y en las papilas dérmicas, hallazgos compatibles con PA. Se suspendió el tratamiento hipoglucemiante oral con vildagliptina, empagliflozina más metformina (comprimido combinado) por indicación de especialista; se inició manejo con insulina, terapia con prednisolona (50 mg) y azatioprina $(50 \mathrm{mg})$ intrahospitalario por tres días donde se evidenció mejoría de las lesiones y finalmente se egresó con igual manejo antihipertensivo que tuvo el paciente, y para el manejo metabólico de la diabetes tipo 2 continuó con metformina y se indicó terapia con insulina de acción prolongada y ultrarrápida; continuó de forma ambulatoria con la terapia inmunosupresora durante un mes y con controles mensuales por médico internista, dermatólogo y alergólogo, con mejoría significativa de las

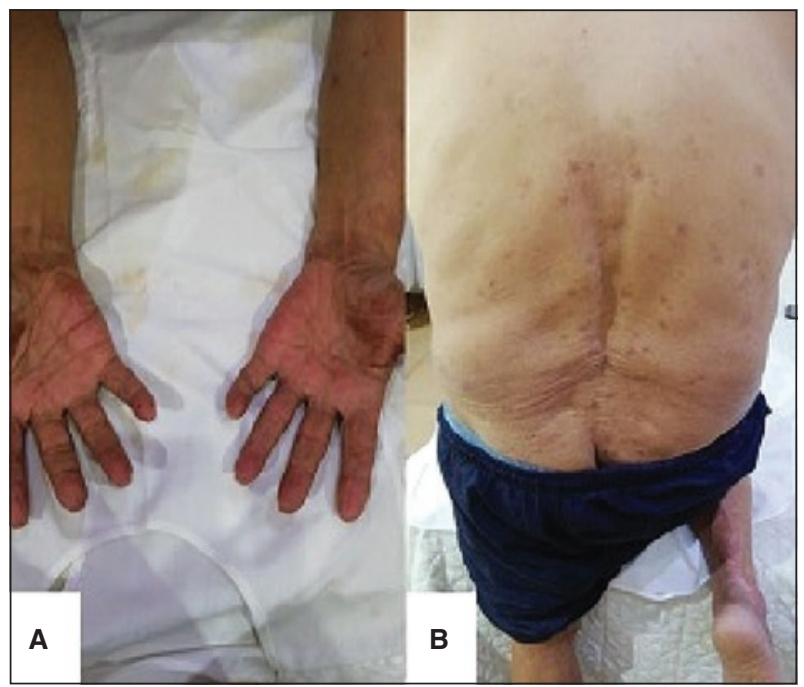

Figura 2. A y B: control fotográfico al 5.0 mes posterior de la terapia farmacológica de inmunomodulación, con mejoría de sus lesiones cutáneas en extremidades, dorso y región lumbar.

lesiones pasados los cinco meses desde la consulta inicial y la terapia medica indicada (Fig. 2).

\section{DISCUSIÓN}

La vildagliptina es un fármaco que inhibe la DPP-4 y que las guías de práctica clínica internacionales y nacionales recomiendan para manejo de pacientes con diabetes tipo 2. Puede administrarse como monoterapia y/o terapia combinada con otros hipoglucemiantes, incluyendo la insulina ${ }^{4}$. Actualmente se desconoce por qué se provoca el PA, pero se plantea que se debe a un desencadenante en pacientes con sensibilidad subyacente, modificando la respuesta inmunitaria o alterando la estructura antigénica de la membrana basal epidérmica ${ }^{5}$.

El PA es una enfermedad ampollosa autoinmune de la cual se desconocen asociaciones genéticas, pero se cree que los autoanticuerpos anti-BP180 juegan un papel importante en la formación de ampollas, si bien el mecanismo patológico mediado por autoanticuerpos ha sido ampliamente estudiado, se sabe poco sobre cómo y por qué la tolerancia inmunitaria a la BP180 puede romperse en ciertos individuos. Las lesiones tienen características vesiculares en grandes extremidades (antebrazo, brazo, mano, 
áreas de flexiones como codos, piernas y muslos) ${ }^{6,7}$. La presentación es variable y puede ser preampollosa, con máculas eritematosas, pápulas, placas urticariforme y lesiones eccematosas ${ }^{8,9}$. La histología presenta características de vesículas, con infiltración moderada y de predominio de eosinófilos, y pueden observarse otras células inflamatorias, sin necrosis de la epidermis, los infiltrados inflamatorios se ubican en la dermis capilar y superficial de la dermis reticular ${ }^{10}$.

Se realizó una revisión de la literatura actual en la cual se han documentado diferentes estudios de PA asociado al uso de iDPP-4, como la investigación de casos y controles de Kridin, et al., donde se documentó que el uso de iDPP-4 tuvo un riesgo tres veces mayor para desarrollar PA (odds ratio [OR]: 3.2; intervalo de confianza del 95\% [IC 95\%]: 1.9-5.4). En el mismo estudio los investigadores describieron las $O R$ ajustadas para vildagliptina y linagliptina: 10.7 (IC 95\%: 5.1-22.4) y 6.7 (IC 95\%: 2.2-19.7), respectivamente $^{2}$. Así mismo, se observan varios reportes de casos como el de Francisco, et al., que refieren tres casos por linagliptina ${ }^{11}$, y se documentó un caso asociado a vildagliptina en un paciente con terapia de reemplazo renal ${ }^{12}$. Skandails, et al. realizaron un estudio en el cual reportaron nueve casos de pacientes con consumo de hipoglucemiantes del grupo de gliptinas ${ }^{13} ; y$ dentro de la detallada revisión literaria que se realizó, el estudio que reportó la mayor frecuencia de casos fue el de Attaway, et al., con 11 casos, los cuales se encontraban en manejo farmacológico hipoglucemiante combinado con vildagliptina más metformina o sitagliptina con metformina con un promedio de tiempo entre 4 y 12 meses $^{14}$.

EI PA es una enfermedad que resulta de una respuesta inmunitaria tipo celular anormal que genera anticuerpos que reaccionan contra el epitelio y el estroma de las hemidesmosomas del epitelio estratificado: antígeno BP230 y antígeno BP180, el primero es una proteína transmembrana con dominio extracelular colágenos y el segundo es una proteína citoplásmica transmembrana tipo II que pertenece a la familia de la plaquina y está involucrado en el anclaje del epitelio estratificado a la membrana basal subyacente. En la piel muchas células expresan DPP-4 (p. ej., linfocitos, monocitos) involucradas en la producción de citocinas, diferenciación tisular y metabolismo del colágeno ${ }^{1}$. El potencial biológico del grupo de fármacos hipoglucemiantes del tipo gliptinas induce modificaciones en la respuesta autoinmune y/o alteraciones antigénicas de la membrana basal de la epidermis llevando al desarrollo del PA; se ha documentado que la inhibición de la DPP-4 genera selección de eosinófilos mediados por CCL11/exotoxina en la dermis y promueve la captación de linfocitos en diferentes enfermedades cutáneas, las proteinasas juegan un papel importante en la transformación y/o destrucción del antígeno BP190, elemento central de la autoinmunidad en la $\mathrm{PA}^{1,5}$.

Esta enfermedad es de difícil diagnóstico al inicio del cuadro clínico, se establece idealmente bajo las manifestaciones clínicas tipo cutáneas y junto con las características histopatológicas, como se realizó en el caso de nuestro paciente. Si bien la descripción histopatológica junto al estudio de inmunofluorescencia directa o indirecta es una herramienta importante en la determinación de la enfermedad, no es necesaria para el diagnóstico de PA (en nuestro paciente no se realizó el estudio de inmunofluorescencia por no contar con la disponibilidad en la institución); así mismo, se encuentra que los estudios de inmunohistoquímicas son relevantes para evidenciar la respuesta de los anticuerpos sobre los antígenos BP180 y BP2307,11 . Es importante resaltar que el diagnóstico de PA en nuestro paciente se fundamentó en las características clínicas de las lesiones y los hallazgos histopatológicos, y que su desarrollo fue posiblemente debido al uso de vildagliptina y no otros medicamentos hipoglucemiantes y/o antihipertensivos, dada la aparición aguda de las lesiones ampollosas secundaria al reciente ingreso del fármaco como tratamiento para la diabetes tipo 2 y a su tiempo de uso, el cual fue menor a 2 meses; así como el riesgo de desarrollar dicho cuadro, como se mencionó anteriormente con la referenciación en investigaciones previas. Dentro del diagnóstico diferencial del PA se encuentran otros tipos de enfermedades ampollosas como la enfermedad autoinmune de inmunoglobulina $A$ en el adulto, dermatitis herpetiforme, eritema polimorfo y pénfigo ${ }^{2,15}$.

El pilar del tratamiento del PA es suprimir la inflamación y la respuesta inmunitaria, para ello se 
podrían utilizar corticosteroides y fármacos inmunosupresores, los cuales han tenido una eficiencia y efectividad alta en la modulación del sistema inmunitario frente a este tipo de patologías. Previo al inicio de la terapia se deben considerar factores asociados con la enfermedad como la extensión y severidad de los síntomas, así como la edad y comorbilidades patológicas, entre ellas las más importantes son la diabetes e hipertensión ${ }^{13,16}$. Dicho tratamiento se puede brindar vía oral o intravenoso si las lesiones son muy severas, en el caso de nuestro paciente se realizó el manejo con prednisolona y azatioprina vía oral, y suspensión de la vildagliptina, con adecuada respuesta clínica de las lesiones cutáneas.

\section{CONCLUSIÓN}

El PA secundario a fármacos continúa siendo una enfermedad poco frecuente en nuestro medio, poseemos poco conocimiento de su epidemiología en el mundo occidental. Según el análisis del reporte del caso, y con la extensa y detallada revisión de la literatura de medicina basada en la evidencia científica, existe una posibilidad elevada de que la vildagliptina haya generado el cuadro clínico de PA en nuestro paciente. No obstante, tenemos claro que dadas las características del tipo de estudio epidemiológico del reporte de caso no se puede llegar a una conclusión de causalidad y para ello es indispensable realizar a futuro un estudio de series de caso y posteriormente un estudio de casos y controles para generar con certeza la probabilidad y la asociación causal de la vildagliptina con el desarrollo del PA como reacción adversa al medicamento (RAM).

Es importante tener en cuenta como clínicos que todos los medicamentos pueden generar una RAM, y que debido al incremento del uso de hipoglucemiantes del tipo gliptinas para pacientes con diabetes tipo 2 para el control glucémico, se termina generando una exposición a los pacientes y que podrían generar este tipo de PA, como se ha reportado en otros estudios epidemiológicos de casos y en nuestro reporte de caso.

\section{FINANCIAMIENTO}

La presente investigación no ha recibido ninguna beca específica de agencias de los sectores público, comercial o sin ánimo de lucro.

CONFLICTO DE INTERESES

Los autores declaran no tener conflictos de intereses.

RESPONSABILIDADES ÉTICAS

Para la realización de la investigación se tuvieron en cuenta los aspectos éticos de la Declaración de Helsinki y la resolución 8430 del Ministerio de Salud de Colombia $^{17,18}$, clasificando esta investigación en un nivel de riesgo mínimo.

Protección de personas y animales. Los autores declaran que para esta investigación no se han realizado experimentos en seres humanos ni en animales.

Confidencialidad de los datos. Los autores declaran que han seguido los protocolos de su centro de trabajo sobre la publicación de datos de pacientes.

Derecho a la privacidad y consentimiento informado. Los autores han obtenido el consentimiento informado de los pacientes y/o sujetos referidos en el artículo.

\section{BIBLIOGRAFÍA}

1. López-Sánchez G, Reyna-Villasmil E. Penfigoide ampolloso inducido por vildagliptina [Internet]. Avan Biomed. 2016;5(1):46-9.

2. Kridin K, Bergman R. Association of bullous pemphigoid with dipeptidyl-peptidase 4 inhibitors in patients with diabetes: Estimating the risk of the new agents and characterizing the patients. JAMA Dermatology. 2018;154(10):1152-8.

3. Lee SG, Lee HJ, Yoon MS, Kim DH. Association of dipeptidyl peptidase 4 inhibitor use with risk of bullous pemphigoid in patients with diabetes. JAMA Dermatology. 2019;155(2):172-7.

4. Pasmatzi E, Monastirli A, Habeos J, Georgiou S, Tsambaos D. Dipeptidyl peptidase-4 inhibitors cause bullous pemphigoid in diabetic patients: Report of two cases. Diabetes Care. 2011;34(8):e133. 
5. Nishie W. Dipeptidyl peptidase IV inhibitor-associated bullous pemphigoid: a recently recognized autoimmune blistering disease with unique clinical, immunological, and genetic characteristics. Immunol Med. 2019;42(1):22-8.

6. Mendonca FMI, Martín-Gutierrez FJ, Ríos-Martín JJ, Camacho-Martinez F. Three cases of bullous pemphigoid associated with dipeptidyl peptidase-4 inhibitors - One due to linagliptin. Dermatology. 2016;232(2):249-53.

7. Aouidad I, Fite C, Marinho E, Deschamps L, Crickx B, Descamps V. A case report of bullous pemphigoid induced by dipeptidyl peptidase-4 inhibitors. JAMA Dermatology. 2013;149(2):243-5.

8. Lee SG, Lee HJ, Yoon MS, Kim DH. Association of dipeptidyl peptidase 4 inhibitor use with risk of bullous pemphigoid in patients with diabetes. JAMA Dermatology. 2019;155(2):172-7.

9. Haber R, Fayad AM, Stephan F, Obeid G, Tomb R. Bullous pemphigoid associated with linagliptin treatment. JAMA Dermatology. 2016;152(2): 224-6.

10. García M, Aranburu MA, Palacios-Zabalza I, Lertxundi U, Aguirre C. Dipeptidyl peptidase-IV inhibitors induced bullous pemphigoid: a case report and analysis of cases reported in the European pharmacovigilance database. J Clin Pharm Ther. 2016;41(3):368-70.

11. Keseroglu HO, Taş-Aygar G, Gönül M, Gököz O, Ersoy-Evans S. A case of bullous pemphigoid Induced by vildagliptin. Cutan Ocul Toxicol. 2017; 36(2):201-2.
12. Usami J, Takezawa Y. DPP-4 inhibitor-associated bullous pemphigoid in a hemodialysis patient. Intern Med. 2020;59(4):593.

13. Skandalis K, Spirova M, Gaitanis G, Tsartsarakis A, Bassukas ID. Drug-induced bullous pemphigoid in diabetes mellitus patients receiving dipeptidyl peptidase-IV inhibitors plus metformin. J Eur Acad Dermatology Venereol. 2012;26(2):249-53.

14. Attaway A, Mersfelder TL, Vaishnav S, Baker JK. Bullous pemphigoid associated with dipeptidyl peptidase IV inhibitors. A case report and review of literature. J Dermatol Case Rep. 2014;8(1):24-8.

15. Béné J, Jacobsoone A, Coupe $P$, Auffret $M$, Babai $S$, Hillaire-Buys $D$, et al. Bullous pemphigoid induced by vildagliptin: a report of three cases. Fundam Clin Pharmacol. 2015;29(1):112-4.

16. Nishie W. Dipeptidyl peptidase IV inhibitor-associated bullous pemphigoid: a recently recognized autoimmune blistering disease with unique clinical, immunological, and genetic characteristics. Immunol Med. 2019;42(1):22-8.

17. Di Ruggiero M. Declaración de Helsinki, principios y valores bioéticos en juego en la investigación médica con seres humanos. Rev Colomb Bioet. 2011;6(1):125-44.

18. Ministerio de Salud. Resolución N.º 008430 de 1993, octubre 4, por la cual se establecen las normas científicas, técnicas y administrativas para la investigación en salud. Gobierno de Colombia, Ministerio de Salud; 1993. 